\title{
Time to change the primary outcome of lupus trials
}

\author{
Frederic A Houssiau ${ }^{1,2}$
}

In $A R D$, a group of outstanding investigators report the results of another lupus trial missing its primary endpoint, namely the Phase III CHABLIS-SC study aimed at testing the efficacy of blisibimod, ${ }^{1}$ composed of a tetrameric BAFF/BLyS domain fused to a human IgG1 Fc region. Interestingly, blisibimod displayed unequivocal effects on biomarkers, such as reduction of circulating $\mathrm{B}$ cells, serum immunoglobulin titres or anti-DNA antibodies and increase in complement levels, changes in line with its mode of action and known to correlate with improved clinical outcome. Moreover, although the trial was not intended to demonstrate renal efficacy, an interesting reduction of proteinuria was noticed. This paradox raises the possibility that the failure of CHABLIS-SC stems more from the choice of the primary efficacy endpoint than from the drug itself, the more so as BAFF/ BLyS was proven to be an appropriate target in four previous clinical trials, namely the belimumab BLISS-52, ${ }^{2}$ BLISS$76^{3}$ and BLISS-SC ${ }^{4}$ and the tabalumab ILLUMINATE- $2^{5}$ studies, all showing the same effects on biomarkers and a significant, although modest, clinical efficacy. Of note, while the primary outcome (SRI-6) failed in the CHABLIS-SC trial, a trend $(p=0.056)$ in favour of blisibimod was demonstrated when a modified endpoint was used, which takes into account, besides SRI-6, achievement of a steroid dose reduction between weeks 40 and 52 compared with day 1.

The main goal of this editorial is to propose a ' $U$ loop' in the choice of the primary outcome measure for lupus trials. So far, and quite logically, the stress has been placed on achieving less disease activity, as measured by one of the many existing indices, such as SELENA-SLEDAI, SLEDAI-2K, BILAG, BICLA, ECLAM

\footnotetext{
'Pôle de Rhumatologie, Institut de Recherche Expérimentale et Clinique, Université catholique de Louvain, Brussels, Belgium

${ }^{2}$ Service de Rhumatologie, Cliniques Universitaires Saint-Luc, Brussels, Belgium
}

Correspondence to Professor Frederic A Houssiau, Pôle de Rhumatologie, Institut de Recherche Expérimentale et Clinique, Université catholique de Louvain, Brussels 1200, Belgium;

frederic.houssiau@uclouvain.be or SRI. Tongue in cheek, their numbers somehow indicate that none of them performs so well... My heretical proposal is to use steroid reduction as a pragmatic primary outcome measure, indirectly reflecting improved disease control! I hear you shouting that this is too far-fetched, but this is exactly why an editorial should be written, not just for summarising and contextualising a study.

I anticipate that choosing a low steroid target as primary endpoint will hardly be accepted by regulatory agencies who label drugs based on their proof of clinical efficacy. Yet, it could be argued that a trial would be considered positive if patients assigned the study drug achieve clinical results comparable with patients randomised to the placebo arm (actually a standard of care arm), with less steroid exposure. The minimal clinically meaningful difference could then become a percentage of steroid reduction, for example, $50 \%$, provided this result is sustained during a sufficiently long period of time, for example, 6 months. Actually, this is what the CHABLIS-SC trial reports. Figure 2 of the paper is quite illustrative in this respect: a similar percentage of patients assigned the study drug and the placebo achieved SRI-6 response at week 52 , but the mean daily dose of prednisone was strikingly lower in the blisibimod group, with twice more patients achieving a dose $\leq 7.5 \mathrm{mg} /$ day.

At a first glance, imposing steroid reduction as primary endpoint raises an ethical concern, a potential medical problem and a methodological issue. Regarding the ethics, it is not possible to taper steroids in patients who do not improve. This concern can be solved by foreseeing escape mechanisms, patients unable to comply with a predefined stringent steroid taper being censored as failure and promptly rescued as per good clinical practice. If the study drug is a wonder molecule, it should reduce signs and symptoms despite steroid reduction. Steroid withdrawal symptoms might constitute a medical issue in patients taking steroids on the long term. This said, the proposal is not to stop steroids but to achieve some steroid spare. The target might actually differ for patients taking steroids at screening compared with those in whom steroids have been started for treatment of a flare. The methodological issue deals with a real paradigm shift, namely to switch from analysis of response at a given time point (the timing is yet another debated issue) to regular monitoring of a per protocol steroid tapering, which is applied except if physician's global assessment of disease (PhGA) activity indicates worsening disease. At the bedside, PhGA drives the steroid dose much more than other scores, which are not used in daily practice, nor for assessment of disease activity, neither for treatment decisions. In other words, this pragmatic approach would parallel clinical practice, steroids being reduced except if patient's condition does not improve. Taken together, the steroid regimen should be very strictly controlled, starting with maximum $20 \mathrm{mg}$ predniso(lo)ne/day, promptly tapered over a few days/weeks. Patients would be evaluated on a regular basis and those who fail the tapering regimen would be declared as treatment failure and treated as needed. At the end of the trial, the percentages of patients achieving a predefined steroid target would be compared.

Before my proposal be turned down, I would like to stress the many reasons why such a primary endpoint would be most welcome. First, a drug allowing steroid reduction would be a major step forward from a patient's perspective. Second, since damage accrual in lupus is mainly due to steroid use, ${ }^{6}$ there is little doubt that reducing the cumulative dose of steroids will reduce morbidity and hopefully mortality in the long term. Third, it should be stressed that two recent Phase II lupus trials that included steroid tapering in their co-primary endpoint (together with disease improvement) reached their target! Thus, anti-IFNAR anifrolumab was shown superior to placebo in the Phase II MUSE trial, which used as primary efficacy endpoint a composite of the SLE Responder Index (SRI-4) at week 24 with a sustained reduction in steroids from weeks 12 through 24 ( $<10 \mathrm{mg}$ prednisolone/day and less than or equal to the dose received at week 1$).^{7}$ For the sake of exactness and fairness, the trial would have been conclusive even without the oral steroid taper requirement. In the Phase 2B AURA lupus nephritis trial, voclosporine (VCS), a new calcineurin inhibitor, was tested against placebo on MMF background. A very stringent steroid tapering was made compulsory to be considered as a responder (sustained reduction of prednisolone $\leq 10 \mathrm{mg} /$ day between weeks 
16 and 24, besides specific renal targets, such as a uP:C ratio $\leq 0.5 \mathrm{mg} / \mathrm{mg}$ ). The results speak for themselves with twice more complete renal remission rates in the MMF/VCS combination group compared with MMF. ${ }^{8}$ Such a difference would probably have been missed if the steroid regimen had been left to physician's/ patient's decision and if a steroid target had not been included in the primary endpoint.

In line with these success stories and with the post hoc analyses of the CHABLIS-SC study, my minimal suggestion is to systematically include a steroid target in the primary endpoint to unmask the true efficacy of a study drug. My bold proposal goes a step further, namely to use steroid reduction as primary outcome measure.

\section{Handling editor Josef S Smolen}

Funding The authors have not declared a specific grant for this research from any funding agency in the public, commercial or not-for-profit sectors.

Competing interests None declared.

Patient consent Not required.
Provenance and peer review Commissioned; externally peer reviewed.

(C) Article author(s) (or their employer(s) unless otherwise stated in the text of the article) 2019. All rights reserved. No commercial use is permitted unless otherwise expressly granted.

\section{A) Check for updates}

To cite Houssiau FA. Ann Rheum Dis 2019;78:581-582.

\section{CLinked}

- http://dx.doi.org/10.1136/annrheumdis-2018213032

Ann Rheum Dis 2019;78:581-582.

doi:10.1136/annrheumdis-2018-213788

\section{REFERENCES}

1 Merrill JT, Shanahan WR, Scheinberg M, et al. Phase III trial results with blisibimod, a selective inhibitor of B-cell activating factor, in subjects with systemic lupus erythematosus (SLE): results from a randomised, double-blind, placebo-controlled trial. Ann Rheum Dis 2018;77:883-9.
2 Navarra SV, Guzmán RM, Gallacher AE, et al. Efficacy and safety of belimumab in patients with active systemic lupus erythematosus: a randomised, placebocontrolled, phase 3 trial. Lancet 2011;377:721-31.

3 Furie R, Petri M, Zamani 0, et al. A phase III, randomized, placebo-controlled study of belimumab, a monoclonal antibody that inhibits B lymphocyte stimulator, in patients with systemic lupus erythematosus. Arthritis Rheum 2011;63:3918-30.

4 Stohl W, Schwarting A, Okada M, et al. Efficacy and safety of subcutaneous belimumab in systemic lupus erythematosus: a fifty-two-week randomized, doubleblind, placebo-controlled study. Arthritis Rheumatol 2017;69:1016-27.

5 Merrill JT, van Vollenhoven RF, Buyon JP, et al. Efficacy and safety of subcutaneous tabalumab, a monoclonal antibody to B-cell activating factor, in patients with systemic lupus erythematosus: results from ILLUMINATE-2, a 52-week, phase III, multicentre, randomised, double-blind, placebo-controlled study. Ann Rheum Dis 2016;75:332-40.

6 Petri M, Purvey S, Fang $H$, et al. Predictors of organ damage in systemic lupus erythematosus: the Hopkins Lupus Cohort. Arthritis Rheum 2012;64:4021-8.

7 Furie R, Khamashta M, Merrill JT, et al. Anifrolumab, an anti-interferon- $\alpha$ receptor monoclonal antibody, in moderate-to-severe systemic lupus erythematosus. Arthritis Rheumatol 2017;69:376-86.

8 Dobronravov V, et al. 48-week complete remission of active lupus nephritis with voclosporin. EULAR Annual Congress 2017. abstract LB0002. 